\title{
Design of Low Pass Fir Filter Using Rectangular, Bartlett and Blackman-Harris Window Techniques
}

\author{
Sriti Pandey ${ }^{1}$, Shalu Choudhary ${ }^{2}$, Pranay Kumar Rahi ${ }^{3}$ \\ B.E. Scholar, Department of Electrical and Electronics Engineering, Institute of Technology Korba, India ${ }^{1,2}$
}

Assistant Professor, Department of Electrical and Electronics Engineering, Institute of Technology Korba, India ${ }^{3}$

\begin{abstract}
The most powerful digital filters are described and compared here. Here various windows are compared and demonstration of the best window is done, which is the one with minimum side lobes. This is hence the major objective of filtering operation. The experimental results show that the FIR filters designed in this paper are effective for filtering operation. Through this paper the intricacies of the window methods are explained in a simple and a subtle manner. In this paper, the design techniques of Low Pass filter using Rectangular Window, Bartlett window and Blackman-Harris of order 10 are presented. On the basis of their performances, it is shown that filter design by using Blackman-Harris window technique is better as it provides better result in terms of magnitude and phase response. The analysis of magnitude and phase response of proposed FIR Low Pass filter is performed using MATLAB simulation.
\end{abstract}

Keywords: DSP, Digital Filter, Low-Pass, FIR, Rectangular Window, Bartlett Window and Blackman-Harris Window.

\section{INTRODUCTION}

Digital filter is essentially a system or network that improves the quality of a signal and/or extracts information from the signals or separates two or more signals which are previously combined. Digital filters are used in numerous applications e.g. control system, system for audio and video processing and communication systems [1]. Digital signal processing is the technique employed for the mathematical manipulation of an information signal so as to modify or improve it. For this purpose filters are mainly used. The filter is used to describe a linear time - invariant system used to perform spectral shaping or frequency-selective filtering. Filter is used in digital signal processing is widely used in a number of ways, such as removal of undesirable noise from desired signals, spectral shaping such as equalization of communication channels, signal detection in radar, sonar and communications, and for performing analysis of the spectra of a variety of signals[2]. Digital filters can be applied to very low frequency signals, such as those occurring in biomedical and seismic applications very efficiently. In addition, the characteristics of digital filters can be changed or a adapted by simply changing the content of a finite number of registers, thus multiple filters are usually used to discriminate a frequency or a band of frequencies from a given signals which is normally a mixture of both desired and undesired signals. These are mainly two types of filter algorithms [1].The world of science and engineering is filled with signals: images from remote space probes, voltages generated by the heart and the brain, radar and sonar echoes, seismic vibrations and countless other applications. Digital signal processing is the science of using computers to understand these types of data [3]. They are finite impulse response filter (FIR) and infinite impulse response filter (IIR). In case of a FIR filter, the response due to an impulse input will decay with in a finite time. But for IIR filter, the impulse response never dies out. FIR filters are commonly known as nonrecursive filters and IIR filters are known as recursive filters. These names came from the nature of algorithm used for these filters. A finite impulse response (FIR) digital filter is one whose impulse response is of finite duration. The impulse response is "finite" because there is no feedback in the filter if put in an impulse (that is, a single "1" sample followed by many " 0 " samples), zeroes will eventually come out after the "1" sample has made its way in the delay line past the entire coefficient. The structure of these algorithms uses a repetitive delay-andadd format that can be represented as "Direct Form-I Structure" [1]. The word "digital" means discrete-valued and implies the signal has an integer-valued independent variable. Digital information includes numbers and symbols. Computers rely on the digital representation of information to manipulate and transform information. Symbols do not have a numeric value, and each is represented by a unique number [4]. FIR filter require no feedback which means that any rounding errors are not compounded by summed iterations, and this system can be easily implemented. The same relative error occurs in each calculation and is inherently stable, since the output of the filter is sum of finite number of finite multiples of input values. The system is easily designed to be linear phase by making the coefficient sequence symmetric. This property is desired for phase sensitive applications such as data communications, crossover filters and mastering. FIR 
filters are very much used in Digital Signal Processing (DSP) applications, because their characteristics behaviour is in linear phase and the feed forward implementation is useful in building stable high-performance filters [5]. . The $\mathrm{Z}$ transform of $\mathrm{N}$ point FIR filter is given by:

$\mathrm{H}(\mathrm{z})=\sum_{\mathrm{N}-0}^{\mathrm{N}} \mathrm{h}(\mathrm{n}) \mathrm{z}^{-\mathrm{n}}, \mathrm{n}=0,1 \ldots \ldots . \mathrm{N}$

Where $\mathrm{N}$ is the order of the filter which has $(\mathrm{N}+1)$ number of coefficients. $h(n)$ is the filter's impulse response. It is calculated by applying an impulse signal at the input. The values of $h(n)$ will determine the type of the filter e.g. low pass, high pass, band pass etc. the values of $h(n)$ are to be determine in the design process and $\mathrm{N}$ represents the order of the polynomial function. The specification of passband, stop band, and transition band is necessary when designing a frequency-selective filter. In passband, frequencies are required to be passed unattenuated. In stopband, frequencies needs to be passed attenuated. Transition band comprises of the frequencies which are lying between the passband and stopband. Therefore, the whole frequency range is divided into one or even more passbands, stopbands, and transition bands. FIR filters are less affected by finite word length [2].

\section{FIR FILTERS}

FIR Filter systems are those systems for which the unit sample response $h(n)$ has finite number of terms. IIR Filter systems are those systems for which such infinite number of unit sample response terms are to be considered [4]. Finite Impulse Response (FIR) filter have some properties for which the designers prefer FIR filter over the IIR filter.

The impulse response of FIR filter is of finite duration but it will be of infinite duration for IIR filter. The advantages of FIR filter over IIR filter are given below]:

1. FIR filters are stable.

2. FIR filters can be easily designed as for its linear phase.

3. FIR filters, when implemented on a finite word length digital system, are free of limit cycle oscillations.

4. There are various methods are available for designing the FIR filter.

5. FIR filter require no feedback i.e. any rounding error are not compounded by summed iteration and for that it is inherently stable.

6. Impulse response is finite [6].

\section{A. Fir Filters Design}

There are essentially three well-known methods for FIR filter design namely:

(1) The window method

(2) The frequency sampling technique

(3)Optimal Filter Design Method [6].

To design a filter means to select the coefficients such that the system has the specific characteristics. The required characteristics are stated in filter specifications. Most of the time filter specifications refer to the frequency response of the filter [4].
Applications:

Traditionally, most digital filter applications have been limited to audio and high-end image processing. With advances in process technologies and digital signal processing methodologies, digital filters are now costeffective in the IF range and in almost all video markets [8].

1. Noise suppression

(a) Imaging devices (medical, etc)

(b) Biosignals (heart, brain)

(c) Signals stored on analog media (tapes)

2. Enhancement of selected frequency ranges

(a) Equalizers for audio systems (increasing the bass)

(b) Edge enhancement in images

3. Removal or attenuation of selected frequencies

(a) Removing the DC component of a signal

(b) Removing interferences at a specific frequency, for example those caused by power supplies

4. Bandwidth limiting

(a) Anti-aliasing filters for sampling

(b) Ensuring that a transmitted signal occupies only its allotted frequency band.

5. Special operations

(a) Differentiation

(b) Integration

(c) Hilbert transforms

6. Simulation/Modelling

(a) Simulating communication channels

(b) Modelling human auditory system [3].

\section{WINDOW TECHNIQUES}

The window design method is first designs an ideal IIR filter and then truncates the infinite impulse response by multiplying it with a finite length window function. The result is a finite-impulse response filter whose frequency response is modified from that of the IIR filter. Multiplying the infinite impulse by the window function in the time domain results in the frequency response of the IIR being convolved with the frequency response of the window function [7]. The two rudimentary factors that describe a window function are:

1) Width of the main lobe (i.e., at what frequency bin is the power half that of the maximum response)

2) Attenuation of the side lobes (i.e., how far away down are the side lobes from the main lobe). This explains about the spectral leakage in the window [2].

The desired frequency response of any digital filter is periodic in frequency and can be expanded in a Fourier series;

$$
\begin{gathered}
\mathbf{H}_{d}\left(\mathbf{e}^{\mathrm{j} \omega}\right)=\sum_{n=-\infty}^{\infty} \mathbf{h}_{d}(\mathbf{n}) \mathbf{e}^{\mathrm{j} \omega} \ldots \ldots \ldots \ldots \\
\text { Where, } \mathbf{h}(\mathbf{n})=\frac{1}{2 \pi} \int_{0}^{2 \pi} \boldsymbol{H}\left(\boldsymbol{e}^{\mathrm{j} \omega}\right) \mathbf{e}^{\mathrm{j} \omega} \mathrm{d} \omega .
\end{gathered}
$$


The Fourier coefficient of the series $h(n)$ are identical to the impulse response of a digital filter. There are two difficulties with the implementation of above equation for designing a digital filter. First, the impulse response is of infinite duration and second, the filter is non-causal and unrealizable. No finite amount of delay can make the impulse response realizable. Hence the filter resulting from a Fourier series representation of $\mathrm{H}()$ is an unrealizable IIR filter [1]. A window is a finite array consisting of coefficients selected to satisfy the desirable requirements. While designing digital FIR filter using window function it is necessary to specify a window function to be used and the filter order according to the required specifications (selectivity and stop band attenuation). These two requirements are interrelated [10]. Window Technique implicates a function called window Function. It is also known as Tapering Function. This report deals with some of the techniques used to design FIR filters. In the beginning the windowing method and the frequency sampling method are discussed in details with their merits and demerits [9]. The windows used in this paper to design the FIR are:

1. Rectangular Window

2. Bartlett window

3. Blackman Harris Window

The various parameters are as follows:

\begin{tabular}{|c|c|c|}
\hline Sr. No. & Parameter & Values \\
\hline 1. & Sampling frequency $(\mathrm{Fs})$ & $48000 \mathrm{~Hz}$ \\
\hline 2. & Cutoff frequency $(\mathrm{Fc})$ & $10800 \mathrm{~Hz}$ \\
\hline 3. & Order $(\mathrm{N})$ & 10 \\
\hline
\end{tabular}

B. Rectangular Window

The rectangular window (sometimes known as the Boxer or Dirichlet window) is the simplest window, equivalent to replacing all but $\mathrm{N}$ values of a data sequence by zeros, making it appear as though the waveform suddenly turns on and off [1] :

$$
\omega_{R}(n) \triangleq\left\{\begin{array}{c}
1,\left(-\frac{M-1}{2}\right) \leq n \leq \frac{M-1}{2} \\
0, \text { otherwise }
\end{array}\right.
$$

Where $\mathrm{M}$ is the window length in samples. The Rectangular Window is what you would obtain if you were to simply segment of finite portion of the impulse response without any shaping in the time domain [4].

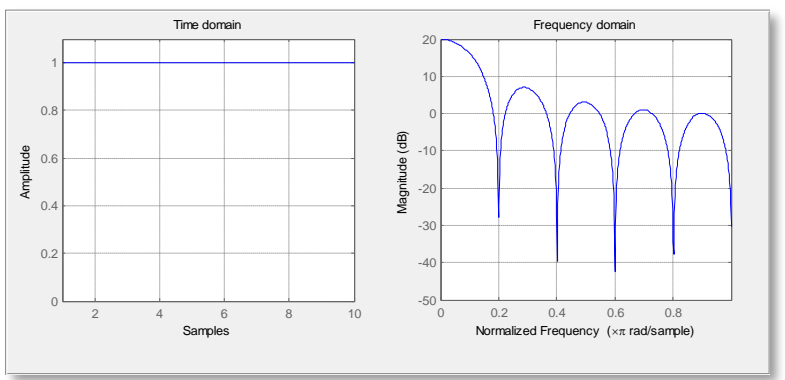

Fig. 1 Time Domain and Frequency Domain of Rectangular Window

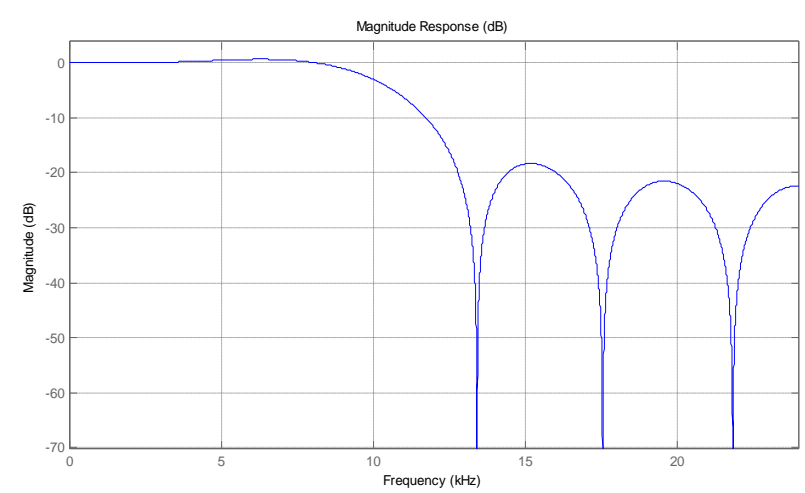

Fig. 2 Magnitude Response of Rectangular Window

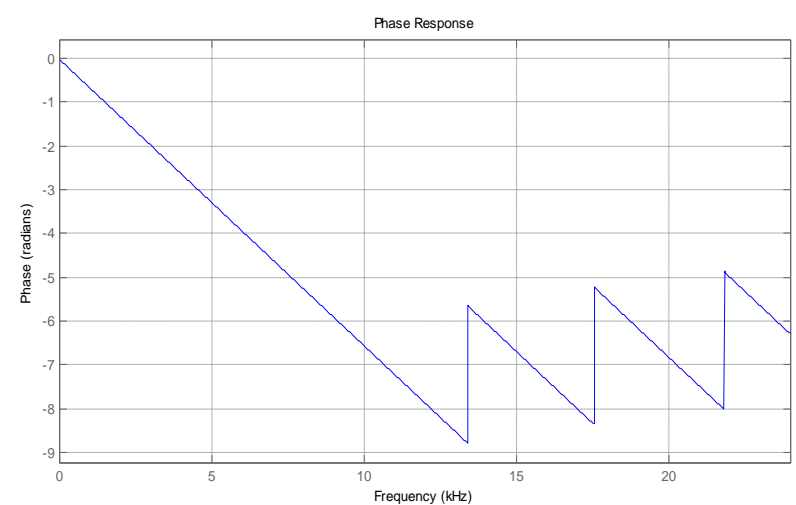

Fig. 3 Phase Response of Rectangular Window

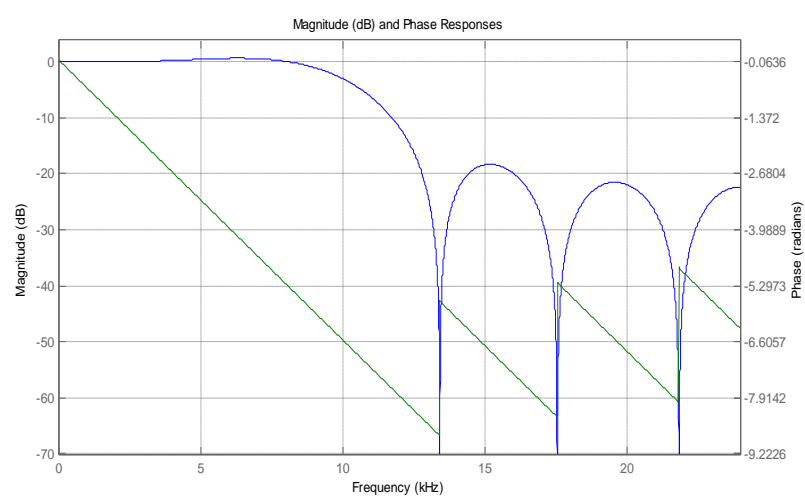

Fig. 4 Magnitude (dB) and Phase Response of Rectangular Window

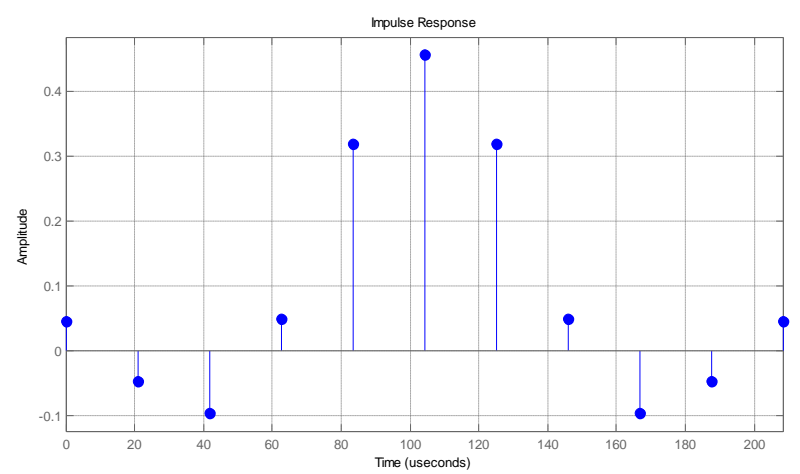

Fig. 5 Impulse Response of Rectangular Window 


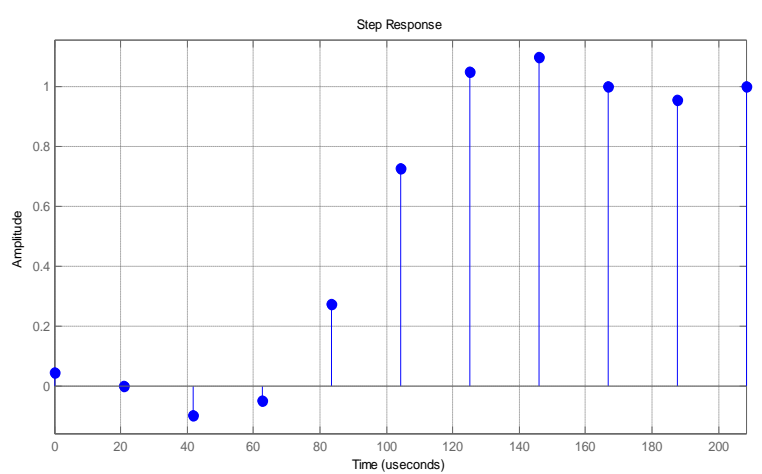

Fig. 6 Step Response of Rectangular Window

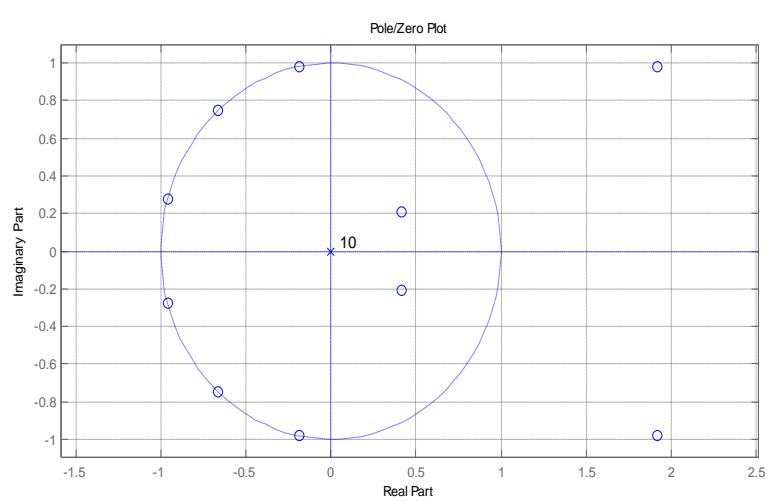

Fig. 7 Pole Zero Plot of Rectangular Window

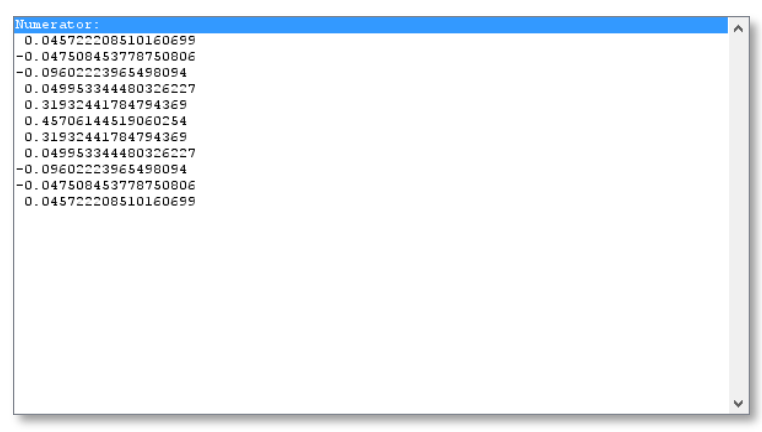

Fig. 8 Filter Coefficient of Rectangular Window

\section{Bartlett Triangular Window}

The Bartlett window is triangularly shaped. Because the Bartlett window can be thought of having been obtained by convolving two rectangular windows of half the width, its transform is easily squaring the transform of the rectangular windows. The Bartlett window has a wider main lobe than the rectangular window, but more attenuated side lobe [4]. The Bartlett window (or simply triangular window) may be defined by;

$$
\boldsymbol{\omega}(\boldsymbol{n})=\boldsymbol{\omega}_{\mathrm{R}}(\boldsymbol{n})\left[1-\frac{|n|}{\frac{M-1}{2}}\right], \boldsymbol{n} \in\left[-\frac{M-1}{2}, \frac{M-1}{2}\right]
$$

And the corresponding transform is [10];

$$
\mathrm{W}(\omega)=\left(\frac{M-1}{2}\right)^{2} a \sin c^{2} \frac{M-1}{2}(\omega)
$$
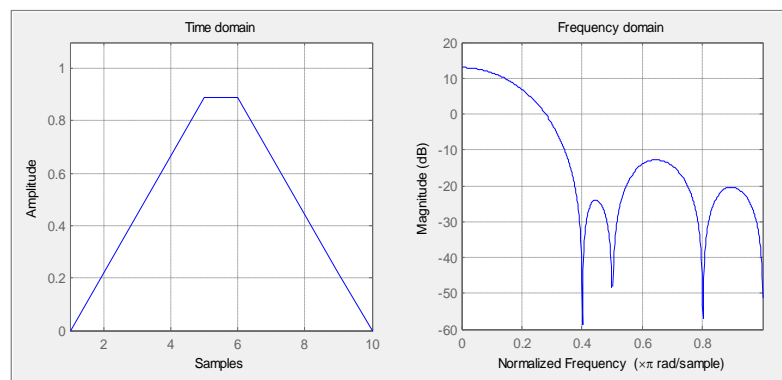

Fig. 9 Time Domain and Frequency Domain of Bartlett Window

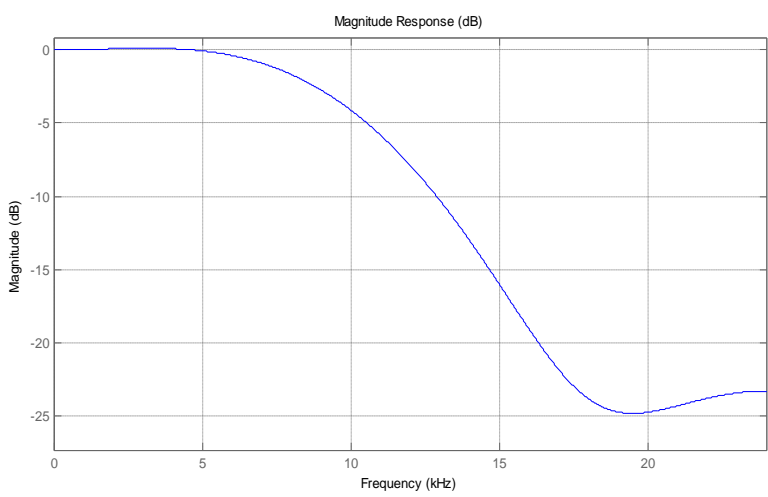

Fig. 10 Magnitude Response of Bartlett Window

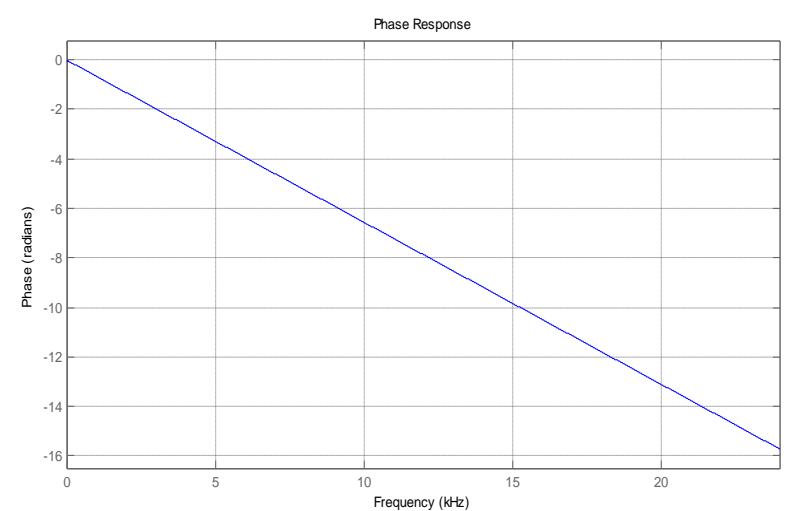

Fig. 11 Phase Response of Bartlett Window

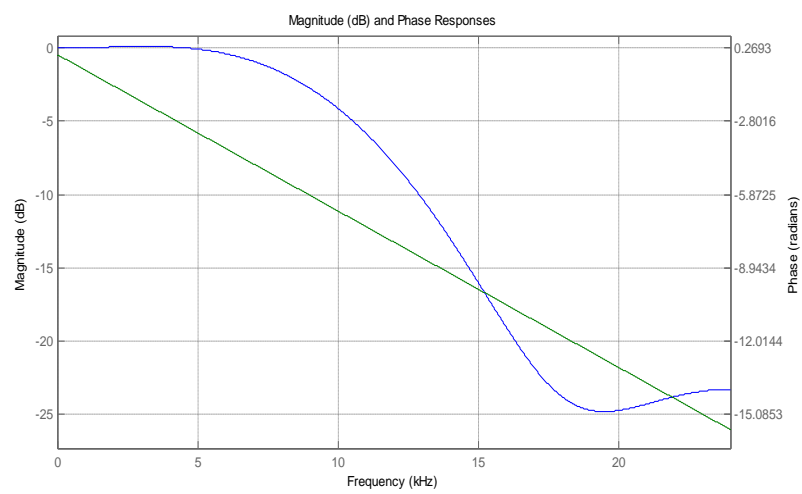

Fig. 12 Magnitude (dB) and Phase Response of Bartlett Window 


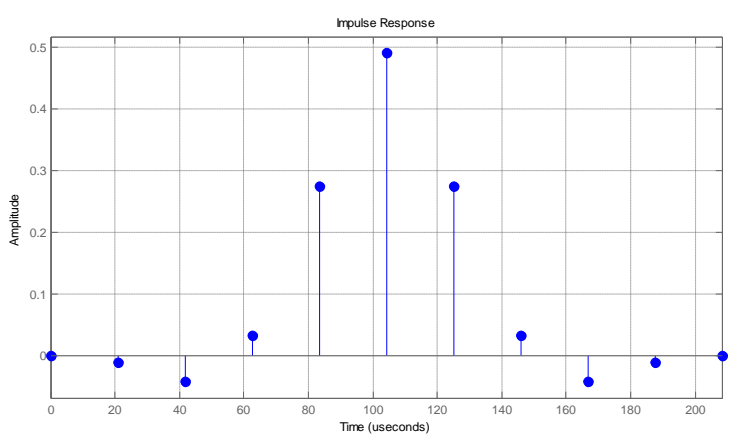

Fig. 13 Impulse Response of Bartlett Window

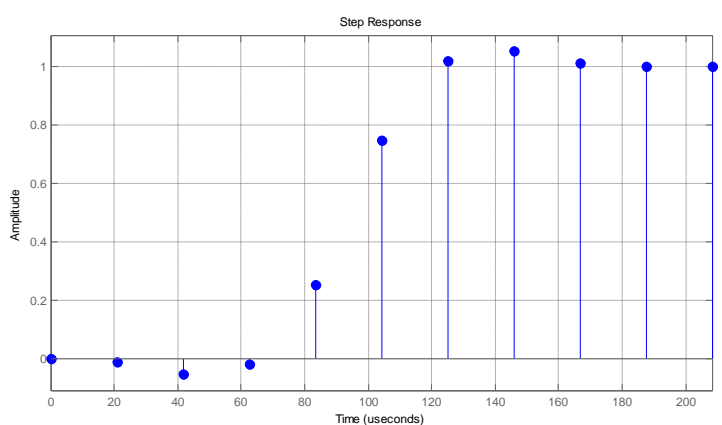

Fig. 14 Step Response of Bartlett Window

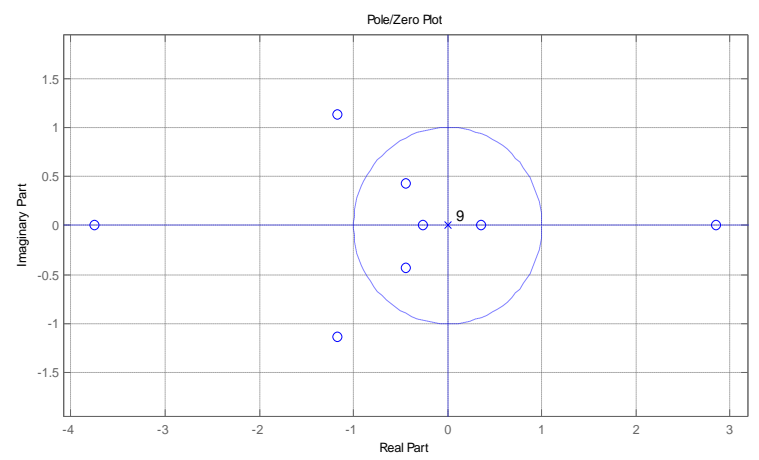

Fig. 15 Pole Zero Plot of Bartlett Window

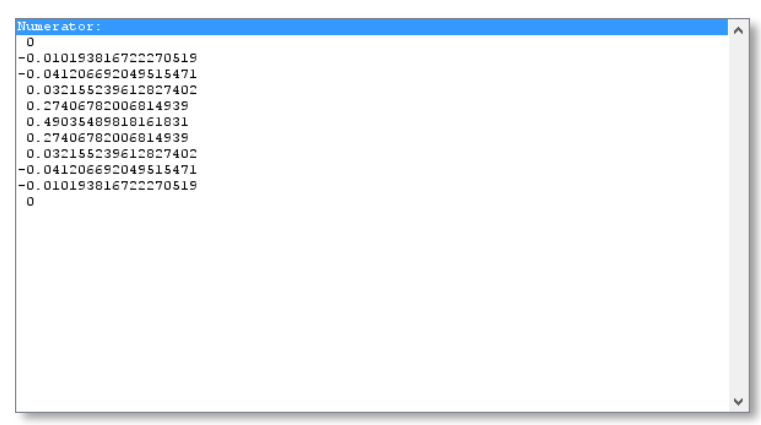

Fig. 16 Filter Coefficient of Bartlett Window

\section{Blackman Harris Window}

Blackman-Harris window is a higher-order generalized cosine window. The Blackman-Harris windows form a family of three and four term windows. The variation on the coefficient allows a compromise between main-lobe width and side-lobe level.
The Blackman-Harris window has one degree of freedom which is used to minimize the level of the side-lobes, and the other is used for the maximization of the roll-off rate. It defines the three-term Blackman-Harris window as the one which uses both degrees of freedom to minimize sidelobe level. The Blackman Harris window has a wider main lobe and a lower minimum side lobe level. The equation for the periodic 4-term Blackman-Harris window of length $\mathrm{N}$ is;

$$
\omega_{\mathrm{n}}=\mathrm{a}_{0}-\mathrm{a}_{1} \cos \left(\frac{2 \pi n}{N-1}\right)+\mathrm{a}_{2} \cos \left(\frac{4 \pi n}{n-1}\right)-\mathrm{a}_{3} \cos \left(\frac{6 \pi n}{N-1}\right)
$$

The periodic window is $\mathrm{N}$-periodic. The following table lists the coefficients [2], [9], [11]:

\begin{tabular}{|c|c|}
\hline Coefficient & Values \\
\hline $\mathrm{a}_{0}$ & 0.35875 \\
\hline $\mathrm{a}_{1}$ & 0.48829 \\
\hline $\mathrm{a}_{2}$ & 0.14128 \\
\hline $\mathrm{a}_{3}$ & 0.01168 \\
\hline
\end{tabular}

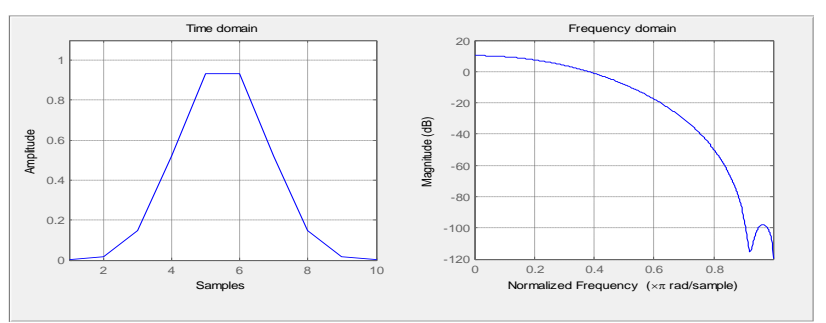

Fig. 17 Time Domain and Frequency Domain of Blackman Harris Window

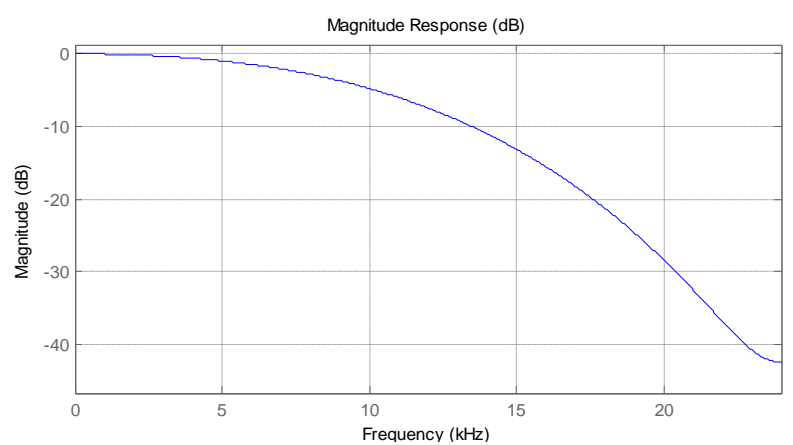

Fig.18 Magnitude Response of Blackman Harris Window

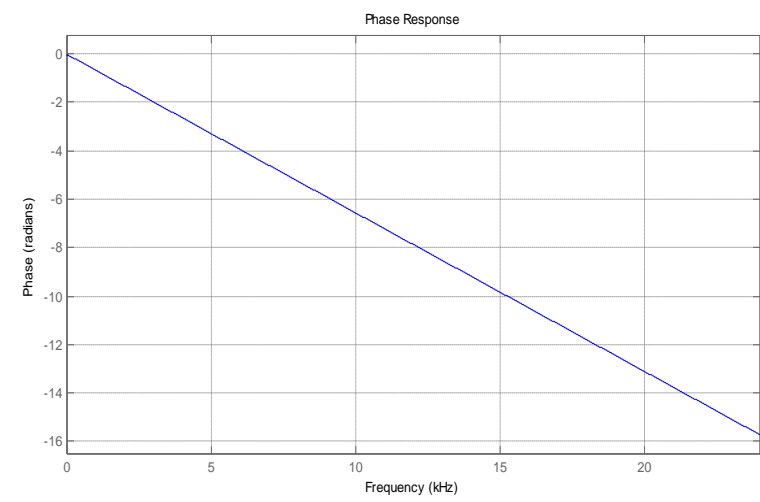

Fig. 19 Phase Response of Blackman Harris Window 


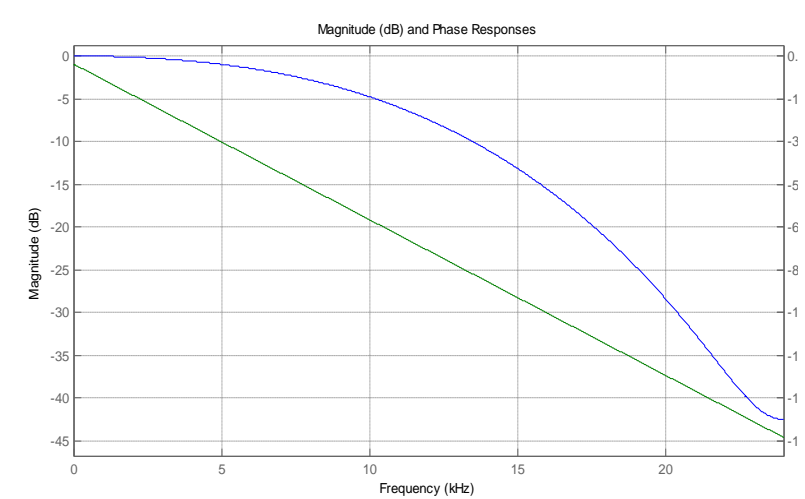

Fig. 20 Magnitude (dB) and Phase Response of Blackman Harris Window

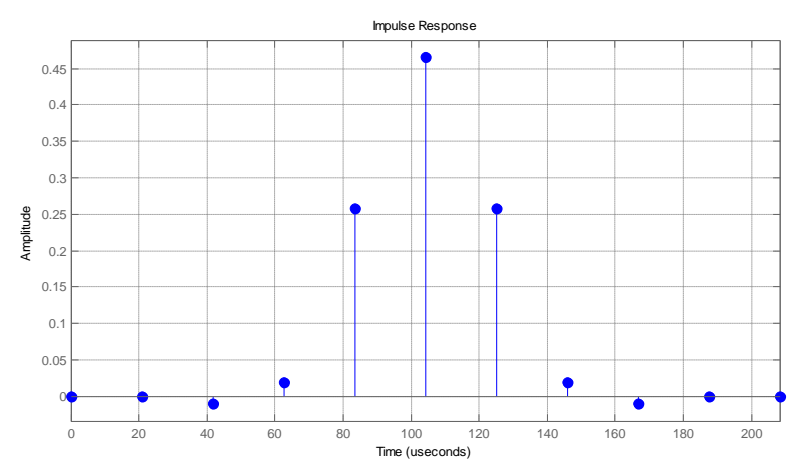

Fig. 21 Impulse Response of Blackman Harris Window

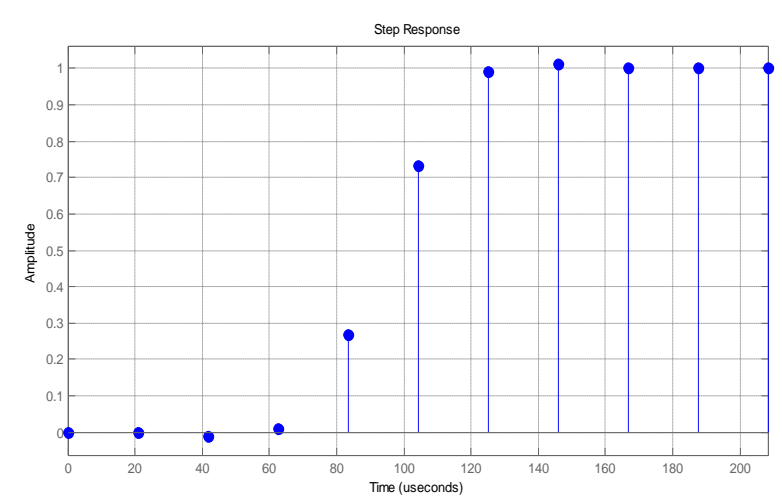

Fig. 22 Step Response of Blackman Harris Window

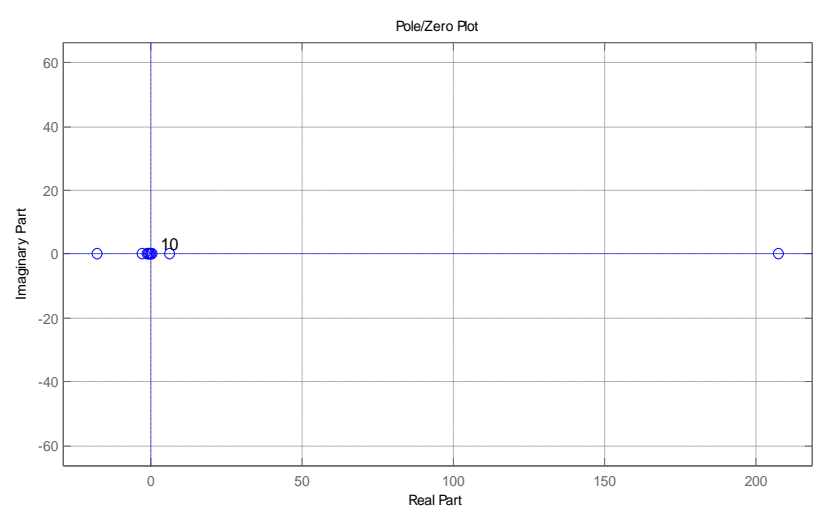

Fig. 23 Pole Zero Plot of Blackman Harris Window

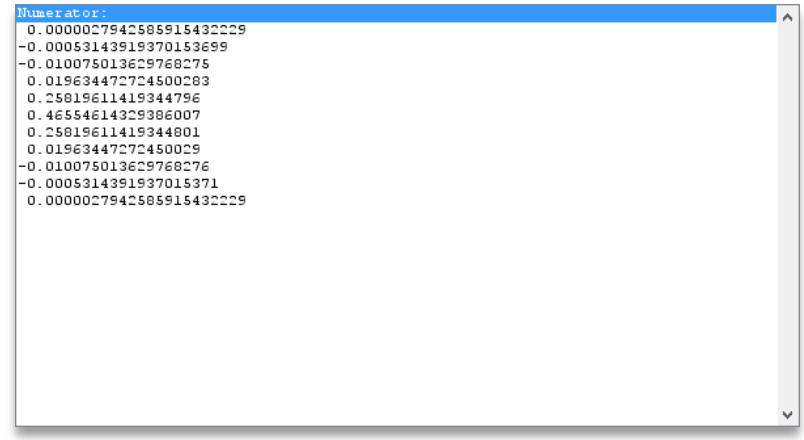

Fig. 24 Filter Coefficient of Blackman Harris Window

D. Comparative Analysis

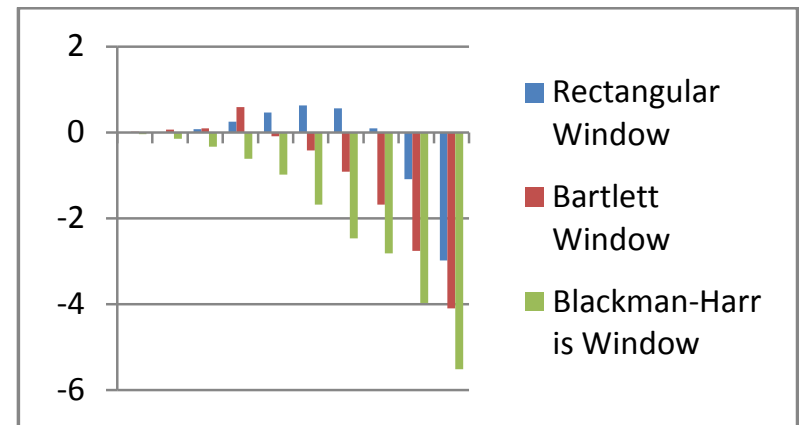

Fig. 25 Magnitude and Frequency Chart of Rectangular, Bartlett and Blackman-Harris Window technique

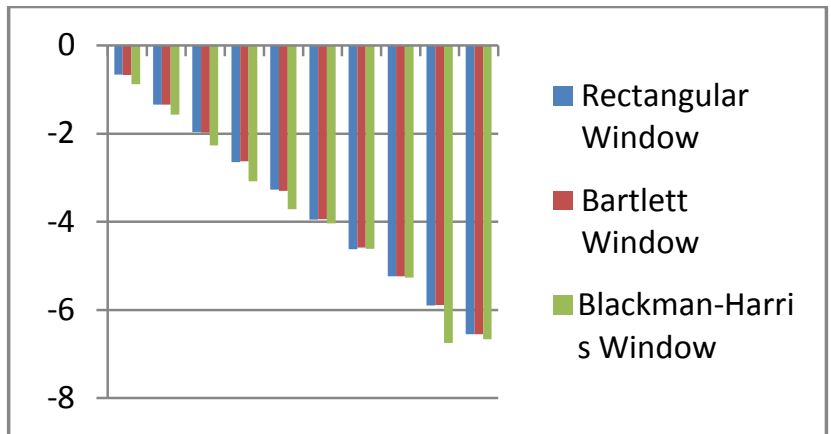

Fig. 26 Phase and Frequency Chart of Rectangular, Bartlett and Blackman-Harris Window technique

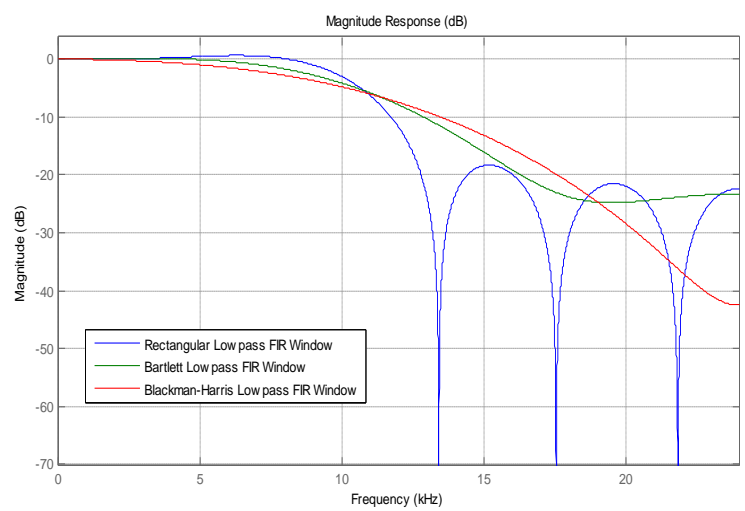

Fig. 27 Magnitude and Frequency Comparison of Rectangular, Bartlett and Blackman Harris Window 


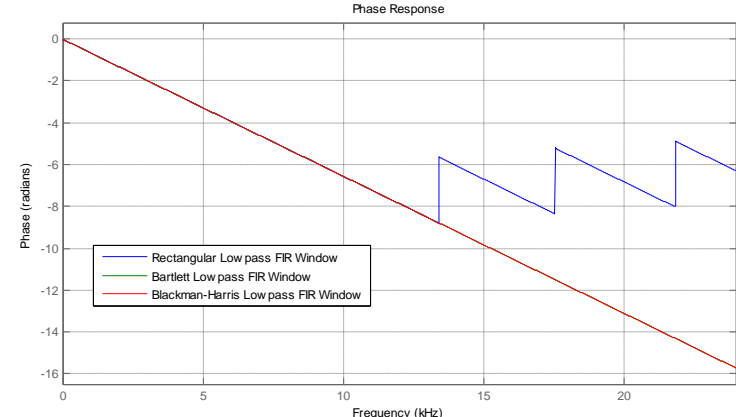

Fig. 28 Phase and Frequency Comparison of Rectangular, Bartlett and Blackman Harris Window

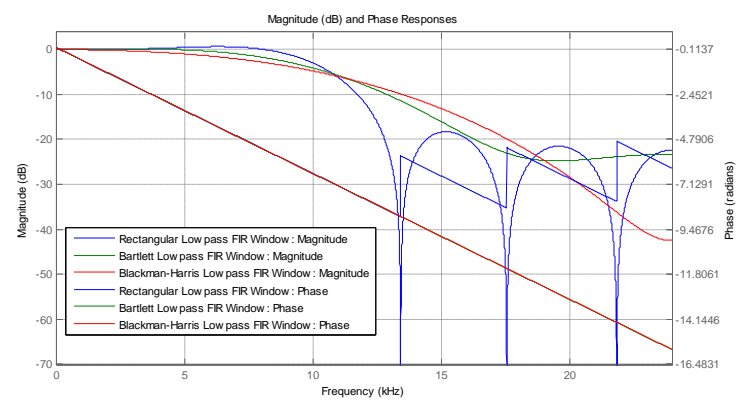

Fig. 29 Magnitude and Phase Response Comparison of Rectangular, Bartlett and Blackman Harris Window

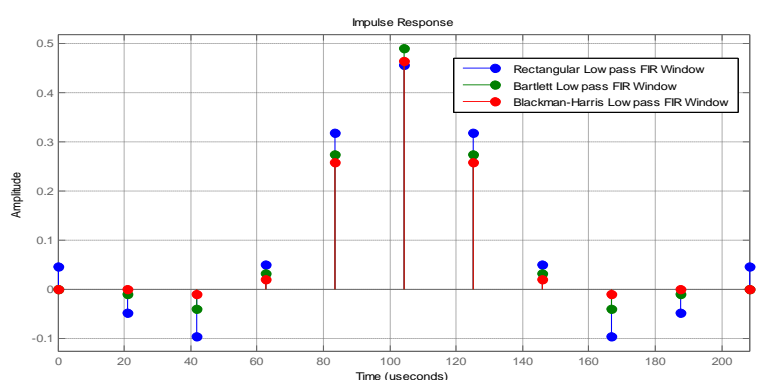

Fig. 30 Impulse Response Comparison of Rectangular, Bartlett and Blackman Harris Window

\begin{tabular}{|c|c|c|c|c|}
\hline $\begin{array}{c}\text { Sr. } \\
\text { No. }\end{array}$ & $\begin{array}{c}\text { Window } \\
\text { Technique }\end{array}$ & $\begin{array}{c}\text { Relative } \\
\text { sidelobe } \\
\text { attenuatio } \\
\text { n }\end{array}$ & $\begin{array}{c}\text { Mainlobe } \\
\text { width(- } \\
\text { 3dB) }\end{array}$ & $\begin{array}{c}\text { Leakage } \\
\text { factor }\end{array}$ \\
\hline 1. & $\begin{array}{c}\text { RECTANGUL } \\
\text { AR }\end{array}$ & $-13 \mathrm{~dB}$ & 0.17188 & $9.2 \%$ \\
\hline 2. & BARTLETT & $-25.8 \mathrm{~dB}$ & 0.28125 & $0.29 \%$ \\
\hline 3. & $\begin{array}{c}\text { BLACKMAN- } \\
\text { HARRIS }\end{array}$ & $-108.2 \mathrm{~dB}$ & 0.41406 & $0 \%$ \\
\hline
\end{tabular}

By using MATLAB 7.12.0 (R2011a) Simulation Technique, we designed all above simulation of Low Pass FIR Filter using RECTANGULAR,BARTELTT \& BLACKMAN HARRIS WINDOW TECHNIQUES. All the above figures shows the difference in their responses in the form of simulation which are time domain \& frequency, magnitude response, phase response, impulse response, step response, pole-zero plot \& filter coefficients.

We performed comparative analysis of the filters, the combined figure of which is shown above.

E. Result

Simulation Result of MATLAB

In Blackman-Harris Window beta value is increased then main lobe width is increased but leakage factor is decreases. When leakage factor is $0.0 \%$ in BlackmanHarris Window then wider main lobe width (0.41406). Interpretation of above table: The table shows parameter specification of windows designing of low pass filters using Rectangular, Bartlett and Blackman-Harris Window. Sampling frequency is $48000 \mathrm{~Hz}$ and cutoff frequency is $10800 \mathrm{~Hz}$. Filter order is 10.

Window Techniques

\begin{tabular}{|c|c|c|c|c|c|c|}
\hline $\begin{array}{c}\text { Frequency } \\
(\mathrm{KHz})\end{array}$ & $\begin{array}{c}\text { Rectangular } \\
\text { Magnitude }\end{array}$ & $\begin{array}{c}\text { Rectangular } \\
\text { Phase }\end{array}$ & $\begin{array}{c}\text { Bartlett } \\
\text { Magnitude }\end{array}$ & $\begin{array}{c}\text { Bartlett } \\
\text { Phase }\end{array}$ & $\begin{array}{c}\text { Blackman- } \\
\text { Harris } \\
\text { Magnitude }\end{array}$ & $\begin{array}{c}\text { Blackman- } \\
\text { Harris } \\
\text { Phase }\end{array}$ \\
\hline 1 & -0.003678 & -0.6596117 & 0.01921499 & -0.6711166 & -0.036712 & -0.882039 \\
\hline 2 & 0.007875 & -1.344151 & 0.06329 & -1.344151 & -0.1500834 & -1.566578 \\
\hline 3 & 0.08209 & -1.965413 & 0.09297 & -1.976918 & -0.33434 & -2.272209 \\
\hline 4 & 0.24797 & -2.644199 & 0.58748 & -2.62886 & -0.6122342 & -3.081384 \\
\hline 5 & 0.46666 & -3.273132 & -0.0859385 & -3.301894 & -0.9772139 & -3.712234 \\
\hline 6 & 0.631135 & -3.955753 & -0.4168438 & -3.942331 & -1.675503 & -4.038204 \\
\hline 7 & 0.565644 & -4.621117 & -0.908658 & -4.584685 & -2.46863 & -4.617282 \\
\hline 8 & 0.0938 & -5.240462 & -1.678435 & -5.23667 & -2.810861 & -5.269224 \\
\hline 9 & -1.091378 & -5.898156 & -2.756072 & -5.890486 & -3.96871 & -6.754345 \\
\hline 10 & -2.979318 & -6.550098 & -4.099772 & -6.553933 & -5.510007 & -6.668981 \\
\hline
\end{tabular}

Magnitude, Phase and Frequency Result of Rectangular, Bartlett and Blackman-Harris Window techniques 


\section{CONCLUSIONS}

The FIR low pass filter has been designed and simulated in this paper using Rectangular, Bartlett and Blackman Harris Window Techniques. These window techniques are thus compared. Digital filters are commonplace and an essential element of everyday electronics. Because the coefficients of digital filters are definite, they can be used to achieve much more complex and selective designs. The results show that the filter design using Blackman-Harris window function has a high main lobe width and sharp transition band compared to Rectangular and Bartlett Window function. It can be seen that the height of the side-lobes is the lowest using the Blackman-Harris. The equivalent noise bandwidth of Blackman-Harris window also reduces as compared to Rectangular Window and Bartlett Window. Hence, among the three windows the Blackman-Harris is considered the best for designing of the low pass filter.

\section{REFERENCES}

[1] Kamlesh Sahu ${ }^{1}$, Ayush Gavel ${ }^{2}$, Pranay Kumar Rahi ${ }^{3},{ }^{1,2}$ BE Scholar,

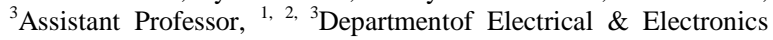
Engineering, Institute of Technology Korba Chhattisgarh, India(Design of Low Pass Fir Filter Using Rectangular, Hanning And Kaiser Window Techniques).

[2] ${ }^{1}$ Mohd. Shariq Mahoob, ${ }^{2}$ Rajesh Mehra, ${ }^{1}$ M.Tech Scholar, ${ }^{2}$ Associate Professor, ${ }^{1,2}$ Department of Electronics and Communication Engineering, National Institute of Technical Teachers' Training \& Research Chandigarh, UT, India(DESIGN OF LOW PASS FIR FILTER USING HAMMING, BLACKMANHARRIS AND TAYLOR WINDOW).

[3] Steven W Smith, "The Scientist and Engineer's guide to Digital Signal Processing", California Technical publishing, San Diego, California, second edition.

[4] ${ }^{1}$ Sriti Pandey, ${ }^{2}$ Kamlesh Sahu, ${ }^{3}$ Pooja Singh Chandel, ${ }^{4}$ Pranay Kumar Rahi; ${ }^{1,2,3}$ B.E.Scholar, 4Assistant Professor, ${ }^{1,2,3,4}$ Department of Electrical \& Electronics Engineering, Institute of Technology Korba, Chhattisgarh, India (LOW PASS FILTER DESIGN AND ANALYSIS USING RECTANGULAR AND BARTLETT WINDOWS).

[5] C. Uthayakumar ${ }^{1}$, and B. Justus $\mathrm{Rabi}^{2},{ }^{1}$ Research Scholar, Karpagam University, Coimbatore, Tamil Nadu, ${ }^{2}$ Professor, Shri Andal Alagar College of Engineering, Mamandur, Tamil Nadu (Design of FIR Filter using Window Method)

[6] Pooja Yadav, Pooja Kumari, Dronacharya College of Engineering, Gurgaon (REVIEW PAPER ON FIR FILTER DESIGN).

[7] Manira Khatun, Assistant Professor, Department of Electronics and Communication Engineering, Mallabhum Institute of Technology, P.S: Bishnupur, Dist: Bankura-722122, W.B, India (IMPLEMENT A NEW WINDOW FUNCTION AND DESIGN FIR FILTERS BY USING THIS NEW WINDOW).

[8] Kajal, Kanchan Gupta, Ashish Saini, Dronacharya College of Engineering (FIR Filter Design using Different Window Techniques).

[9] Vandana Kurrey ${ }^{1}$, Shalu Choudhary ${ }^{2}$, Pranay Kumar Rahi ${ }^{3},{ }^{1,2} \mathrm{BE}$ scholar, ${ }^{3}$ Assistant Professor, ${ }^{1,2,3}$ Department of Electrical and Electronics Engineering, Institute of Technology, Korba, Chhattisgarh, India(A Comparative Performance Analysis of High Pass Filter Using Bartlett Hanning And Blackman Harris Windows ).

[10] Manjinder Kaur ${ }^{1}$, Sangeet Pal Kaur ${ }^{2}$, PG Student, Dept. of ECE, Punjabi University, Patiala, Punjab, India ${ }^{1}$, Assistant Professor, Dept. of ECE, Punjabi University, Patiala, Punjab, India ${ }^{2}$ (FIR Low Pass Filter Designing Using Different Window Functions and their Comparison using MATLAB).

[11] ${ }^{1}$ Suman Mahant, ${ }^{2}$ Manju Kanwar, ${ }^{3}$ Ruchi Baghel, ${ }^{4}$ Pranay Kumar Rahi, ${ }^{1,2,3}$ BE Scholar, ${ }^{4}$ Assistant Professor, ${ }_{1,2,3,4}$ Department of
Electrical \& Electronics Engineering, Institute of Technology, Korba, Chhattisgarh, India(Designing a Low- Pass Fir Digital Filter By Using Bartlett Hanning and Blackman Harris Window Technique).

\section{BIOGRAPHIES}

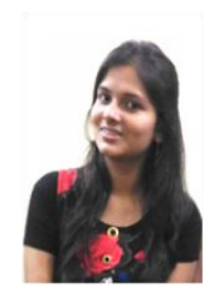

Sriti Pandey pursuing Bachelor of Engineering in Electrical and Electronics Engineering in 6th semester from Institute of Technology Korba, Chhattisgarh Swami Vivekananda Technical University, Chhattisgarh, India.

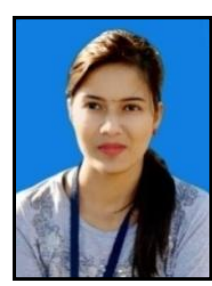

Shalu Choudhary pursuing Bachelor of Engineering in Electrical and Electronics Engineering in 6th semester from Institute of Technology Korba, Chhattisgarh Swami Vivekananda Technical University, Chhattisgarh, India.

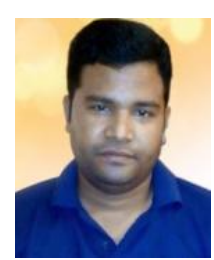

Pranay Kumar Rahi Received the Bachelors of Engineering degree in Electronics and Telecommunication Engineering from Government Engineering College, Guru Ghasidas University, Bilaspur, Chhattisgarh, India in 2004,working as a Assistant Professor in Electrical and Electronic Engineering of Institute of Technology Korba since 2008 and pursuing Masters of Engineering in Electronics and Communication Engineering from National Institute of Technical Teacher's Training\& Research, Punjab University, Chandigarh, India. Presently working as assistant professor in Department of Electrical and Electronic Engineering, Institute of Technology Korba, Chhattisgarh since 2008. He has authored More than 40 research publications and published journal papers and research papers in the leading International and National journal. His primary research interest includes Digital Signal Processing, VLSI Design, Control Systems and Digital Electronics and logic design. 